\title{
Cancer Screening in Patients with Unprovoked Thromboembolism: How to do it and Who Benefits?
}

\author{
Filipa Ferreira ${ }^{1}$, José Pereira ${ }^{1}$, Ana Lynce ${ }^{2}$, José Nunes Marques ${ }^{1}$, Ana Martins ${ }^{1}$ \\ 1. Medical Oncology, Centro Hospitalar de Lisboa Ocidental, Lisbon, PRT 2. Internal Medicine, Centro Hospitalar de \\ Lisboa Ocidental, Lisbon, PRT
}

Corresponding author: Filipa Ferreira, fi_lipaferreira@hotmail.com

\begin{abstract}
Introduction: Unprovoked venous thromboembolism (uVTE) may be the first manifestation of cancer. The main objectives of this study were to compare limited screening (LS) and extended screening (ES) and to make a protocol to approach these patients.

Methods: This is a retrospective, unicentric observational study that included 245 patients with venous thromboembolism (VTE) admitted to an Internal Medicine Service for five years. The incidence of cancer and mortality during hospitalization, and at one and three years after admission were calculated in both LS and ES groups and compared.

Results: Of the 245 patients with VTE, 59 (24.1\%) had UVTE: 35 (59.3\%) were submitted to LS and 24 (40.7\%) to ES, with 10 (4.1\%) diagnosis of cancer. In the following three years, 10 more patients were diagnosed. There were no statistically significant differences in inpatient diagnosis rates (8.6\% vs. $4.2 \%$; $\mathrm{p}=0.51)$ or inhospital mortality ( $2.9 \%$ vs. $4.2 \%$; $=0.79$ ) or mortality at one year $(8.6 \%$ vs. $8.3 \%$; $=0.97)$ and three years ( $20.0 \%$ vs. $20.8 \%$; $=0.94$ ) between LS and ES groups respectively. The Computerized Registry of Patients with Venous Thromboembolism (RIETE) score was equal or superior to 3 in $69.5 \%(\mathrm{~N}=41)$ of the population with uVTE.
\end{abstract}

Discussion: The results of our study are consistent with the literature; there are no differences between screenings, as the difference in the number of diagnoses does not reflect on mortality.

Conclusion: There were no statistically significant differences between the two types of screening in this population. We suggest a protocol that includes the RIETE score to better select the patients who might benefit the most from an ES.

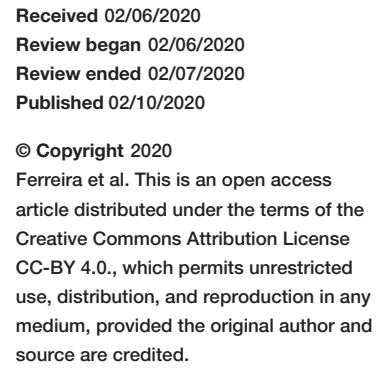

began $02 / 06 / 2020$ article distributed under the terms of the CCative Commons Attribution License use, distribution, and reproduction in any source are credited.
Categories: Internal Medicine, Miscellaneous, Oncology

Keywords: unprovoked thromboembolism, cancer screening, thromboembolism, cancer, mortality

\section{Introduction}

Cancer is a well-established independent risk factor for venous thromboembolism (VTE), and an episode of unprovoked VTE (UVTE) may be the first manifestation of a hidden neoplasm [1-3]. The incidence of cancer associated with uVTE is about $4.5 \%-5 \%$, with the risk being particularly high in the first six months after the episode and higher in colorectal, pancreatic, brain and lung tumours [3-6]. Most studies show a risk reduction after the first 12 months, equalling the general population, but others show increased rates up to six years $[4,5,7]$.

Cancer screening after an episode of UVTE may allow an early diagnosis of occult cancer. However, the investigation of patients with uVTE lacks national or international guidelines that clarify what types of tests should be done and in which patients. Several trials compare two types of screening: limited and extended. Limited screening (LS) strategies are variable in the literature, but usually include clinical history, physical examination, general analyses (blood count, kidney function, ionogram, and liver function) and chest X-ray; some trials also include screening tests related to the patient's age and gender [1,5]. Besides limited screening examinations, extended screening (ES) includes additional tests such as ultrasound, computed tomography (CT) or positron emission tomography/CT (PET/CT) [5]. Most trials conclude that LS diagnoses up to about $90 \%$ of occult neoplasms, with a difference in the number of diagnoses between the two types of screening that is not reflected in mortality [5,8-12]. ES is also associated with more false positives, unnecessary research and higher costs [5,12]. The 2012 National Institute for Health Care Excellence (NICE) guidelines suggest that all patients should undergo medical history, physical examination, general analysis, and chest X-ray, and that abdominal-pelvic CT should be performed on patients older than 40 years and mammography in all women [5]. On the other hand, the 2016 guidelines of the Anticoagulation Forum suggest that patients should undergo LS, with clinical history, physical examination, general analysis, chest 
Several clinical and analytical factors have revealed a predictive value for the identification of patients with uVTE at increased risk of cancer [14-17]. The Computerized Registry of Patients with Venous Thromboembolism (RIETE) score was validated for this purpose, with the highest risk being present in patients who meet three or more of the following criteria: male gender, age equal or superior to 70 years, chronic lung disease, anemia, thrombocytosis, previous VTE and recent surgery [18]. However, it remains unclear which patients should undergo more extensive screening and whether the earlier detection of cancer improves patients' morbidity, mortality, and quality of life.

The main objectives of this study are: to compare the two types of screening (LS and ES) through the evaluation of the exams performed on the population with uVTE in an Internal Medicine Service over five years, the calculation of the incidence of cancer (during hospitalization, in the first year and in the second and third years after) and evaluation of its impact on mortality, and depending on the data obtained, to develop a protocol to approach these patients. As secondary objectives, it is also intended to evaluate the characteristics of the various patient subgroups and calculate the RIETE score for the study population.

\section{Materials And Methods}

\section{Study design}

This is an observational, retrospective, and single-centre study, which includes patients hospitalized with the diagnosis of VTE in the Internal Medicine Service of Hospital São Francisco Xavier over a period of five years, between January 1, 2011 and December 31, 2015. Patients older than 18 years and diagnosed with VTE according to ICD-9 (International Statistical Classification of Diseases and Related Health Problems 9) were included. The selection of patients was performed using the Homogeneous Diagnostic Groups, a Portuguese classification system of the diagnoses of hospitalized patients in acute hospitals. The following diagnoses and respective codes were considered: 415.1 - Embolism and pulmonary infarction, 415.19 - Pulmonary thromboembolism (PTE), 453 - Embolism and venous thrombosis, not elsewhere classified, 453.2 Embolism and thrombosis of the vena cava, 453.3 - Embolism and thrombosis of the renal vein, 453.4 Embolism and venous thrombosis of unspecified deep vessels of the limbs, 453.5 - Embolism and chronic thrombosis of deep lower extremity vessels, 453.6 - Embolism and thrombus of chronic of superficial vessels of lower extremity, 453.7 - Embolism and chronic thrombosis of specified vessels not classified elsewhere, 453.8 - Embolism and thrombosis of specified veins not elsewhere classified and 453.9 - Embolism of thrombosis of unspecified location.

uVTE was defined as the VTE that occurs in the absence of a previous history of VTE, active neoplasia, coagulopathy, hormonal therapy, pregnancy/puerperium, or surgery, trauma or immobilization in the previous three months. LS was defined as the screening that includes clinical history, physical examination, general analyses (blood count, renal function, ionogram, and liver function), chest X-ray (and/or CT angiography of the chest in case of PTE) and screening appropriate to age and gender (breast examination and/or mammography in women older than 50 years, colpocytology in sexually active women aged between 18 to 70 years; rectal examination and/or prostate specific antigen (PSA) in men older than 40 years; colonoscopy in patients older than 50 years). ES included, besides LS tests, others such as analytic panel of tumour markers, autoimmune analytic profile, analytic study of thrombophilia, ultrasound, CT, nuclear magnetic resonance (NMR), endoscopic exams or other invasive examinations.

Data collection was carried out by consulting the hospital clinical process through the Portuguese platform SAM (Sistema de Apoio ao Médico - Physician Support System) and extra-hospital through the Portuguese RSE (Registo de Saúde Eletrónico - Electronic Health Record). The variables collected were: gender; birth date; characteristics of the thromboembolic phenomenon (PTE and/or deep vein thrombosis (DVT)); comorbidities, including the existence of previous VTE and a known neoplasm (as well as its characteristics primary tumour, stage, active treatment); diagnosis of cancer during hospitalization (tests performed, primary tumour, stage); anticoagulant therapy (inpatient and outpatient); diagnosis of cancer in the following year and 2-3 years thereafter (primary tumour, stage); mortality of patients diagnosed with cancer (inpatient and after discharge).

\section{Statistical analysis}

Variables of a continuous nature are presented as mean ( \pm standard deviation) or median (interquartile range), depending on the underlying distribution of the data. Variables of a discrete nature are presented as absolute and relative frequencies. The Chi-square test was used to compare categorical variables and the Student's t-test was used to compare continuous variables. Values of $\mathrm{p}<0.05$ were considered statistically significant. The data were analysed using Microsoft Excel and Statistical Package for the Social Sciences (SPSS Inc., Chicago, IL), version 25.

\section{Results}

During the period considered, 4538 patients were admitted to the Internal Medicine Service, of which 245 (5.40\%) were diagnosed with VTE. The median age of the patients was 75 years and 88 patients (35.9\%) were 


\section{Cureus}

male. Regarding the embolic phenomenon, most patients ( $\mathrm{N}=138$; 56.3\%) had a diagnosis of PTE, 60 (24.5\%) had DVT and 47 (19.2\%) had both. Among comorbidities, 62.9\% ( $\mathrm{N}=154)$ patients had arterial hypertension, $31.4 \%(\mathrm{~N}=77)$ had dyslipidaemia, $22.0 \%(\mathrm{~N}=54)$ had diabetes, $12.7 \%(\mathrm{~N}=31)$ had a previous episode of VTE

(13 of these still under therapeutic anticoagulation) and $25.7 \%(\mathrm{~N}=63)$ had a previous diagnosis of cancer. Of the patients with known cancer, the majority had gastrointestinal (31.7\%; $\mathrm{N}=20)$, genitourinary $(22.2 \%$, $\mathrm{N}=14)$ or breast $(14.3 \% ; \mathrm{N}=9)$ tumours, with $41.3 \%$ in stage IV and $65.1 \%$ with no active treatment at the time of admission. The characteristics of the general population can be seen in Table 1 and those of the subgroup with known diagnosis of cancer in Table 2.

\begin{tabular}{|c|c|c|}
\hline \multicolumn{3}{|l|}{ General Population (N=245) } \\
\hline \multirow{2}{*}{ Gender $(\mathbb{N} ; \%)$} & Male & $88(35.9 \%)$ \\
\hline & Female & $157(51.8 \%)$ \\
\hline \multicolumn{2}{|l|}{ Median age (years) [Min;:Max] } & $75[18 ; 98]$ \\
\hline \multirow{3}{*}{$\operatorname{VTE}\left(N_{*} ; \%\right)$} & Pulmonary thromboembolism & $138(56.3 \%)$ \\
\hline & Deep vein thrombosis & $60(24.5 \%)$ \\
\hline & Both & $47(19.2 \%)$ \\
\hline \multirow{13}{*}{ Comorbidities $(\mathbb{N} ; \%)$} & Arterial hypertension & $154(62.9 \%)$ \\
\hline & Diabetes & $54(22.0 \%)$ \\
\hline & Chronic lung disease & $24(9.8 \%)$ \\
\hline & Coronary disease & $14(5.7 \%)$ \\
\hline & Dyslipidaemia & $77(31.4 \%)$ \\
\hline & obesity & $35(14.3 \%)$ \\
\hline & Coagulopaties & $5(2.0 \%)$ \\
\hline & Cancer & $63(25.7 \%)$ \\
\hline & Tabagism & $34(13.9 \%)$ \\
\hline & Alcoolism & $13(4.3 \%)$ \\
\hline & Surgery, trauma or immobilization in the last 3 months & $29(11.8 \%)$ \\
\hline & Pregnancy or puerperium & $2(0.8 \%)$ \\
\hline & Hormonal therapy & $2(0.8 \%)$ \\
\hline \multirow{5}{*}{ Previous VTE (N; \%) } & Total & $31(12.7 \%)$ \\
\hline & $<6$ months & $9(3.7 \%)$ \\
\hline & $\geq 6$ months & $12(4.9 \%)$ \\
\hline & Unknown & $10(4.1 \%)$ \\
\hline & Under anticoagulation & $13(4.3 \%)$ \\
\hline \multirow{9}{*}{ Anticoagulation (N; \%) } & Inpatient & \\
\hline & Low-molecular-weight heparin & $196(80.0 \%)$ \\
\hline & Low-molecular-weight heparin with bridge to Warrarin & $31(12.7 \%)$ \\
\hline & Low-molecular-weight heparin with bridge to Direct Oral Anticoagulants & $8(3.3 \%)$ \\
\hline & Thrombolysis followed by Low-molecular-weight heparin & $10(4.1 \%)$ \\
\hline & Outpatient & \\
\hline & Low-molecular-weight heparin & $62(25.3 \%)$ \\
\hline & Warfarin & $97(39.6 \%)$ \\
\hline & Direct Oral Anticoagulants & $52(21.2 \%)$ \\
\hline
\end{tabular}




\section{Cureus}

\begin{tabular}{|c|c|c|}
\hline & Not instituted for clinical reasons & $14(5.7 \%)$ \\
\hline \multirow{4}{*}{ Mortality during hospitalization $(\mathbb{N} ; \%)$} & Total & $20(8.2 \%)$ \\
\hline & Related to known cancer & $9(3.7 \%)$ \\
\hline & Related to inpatient cancer diagnosis & $2(0.8 \%)$ \\
\hline & Other causes & $9(3.7 \%)$ \\
\hline \multirow{2}{*}{ VTE recurrence } & Total & $18(7.3 \%)$ \\
\hline & Cancer patients & $10(4.1 \%)$ \\
\hline
\end{tabular}

\section{TABLE 1: Characteristics of the general population}

VTE: venous thromboembolism.

Patients with known cancer at admission (N=63)

\begin{tabular}{|c|c|c|}
\hline \multirow{9}{*}{ Primary tumour $(\mathrm{N} ; \%)$} & Gastrointestinal & $20(31.7 \%)$ \\
\hline & Urological & $14(22.2 \%)$ \\
\hline & Breast & $9(14.3 \%)$ \\
\hline & Hematologic & $6(9.5 \%)$ \\
\hline & Gynecological & $4(6.3 \%)$ \\
\hline & Thoracic & $3(4.8 \%)$ \\
\hline & Occult primary & $3(4.8 \%)$ \\
\hline & Central Nervous System & $3(4.8 \%)$ \\
\hline & Soft tissues & $1(1.6 \%)$ \\
\hline \multirow{3}{*}{ Stage (N; \%) } & I-III & $35(55.6 \%)$ \\
\hline & IV & $26(41.3 \%)$ \\
\hline & Unknown & $2(3.2 \%)$ \\
\hline \multirow{5}{*}{ Treatment (N; \%) } & No active treatment & $41(65.1 \%)$ \\
\hline & Under active treatment & $22(34.9 \%)$ \\
\hline & Chemotherapy & $14(22.2 \%)$ \\
\hline & Hormonal therapy & $8(12.7 \%)$ \\
\hline & Central venous catheter & $12(19.0 \%)$ \\
\hline
\end{tabular}

\section{TABLE 2: Characteristics of the group of patients with known cancer at admission}

Analysing the personal history of the patients, accordingly with the definition of uVTE, 186 patients were excluded, since they presented risk factors for provoked VTE. The remaining 59 (24.1\%) did not have any evident risk factors for VTE, whereby they had an episode of UVTE. Of these, 35 (59.3\%) underwent limited screening tests and 24 (40.7\%) underwent ES (Table 3) (Figure 1). 


\section{Cureus}

\begin{tabular}{|ll|}
\hline Tests performed on patients with UVTE (N=59) & \\
\hline Clinical history and physical examination & $59(100 \%)$ \\
\hline General analysis & $59(100 \%)$ \\
\hline Panel of tumor markers & $15(25.4 \%)$ \\
Autoimmune profile and study of thrombophilia & $8(13.6 \%)$ \\
Chest X-Ray (and/or thoracic AngioTC if PTE) & $59(100 \%)$ \\
Screening tests suitable for age and gender & $6(10.2 \%)$ \\
UItrasound & $31(52.5 \%)$ \\
Computed tomography and nuclear magnetic resonance & $7(11.9 \%)$ \\
Endoscopic exams & $3(5.1 \%)$
\end{tabular}

TABLE 3: Tests performed on patients with unprovoked venous thromboembolism (UVTE)

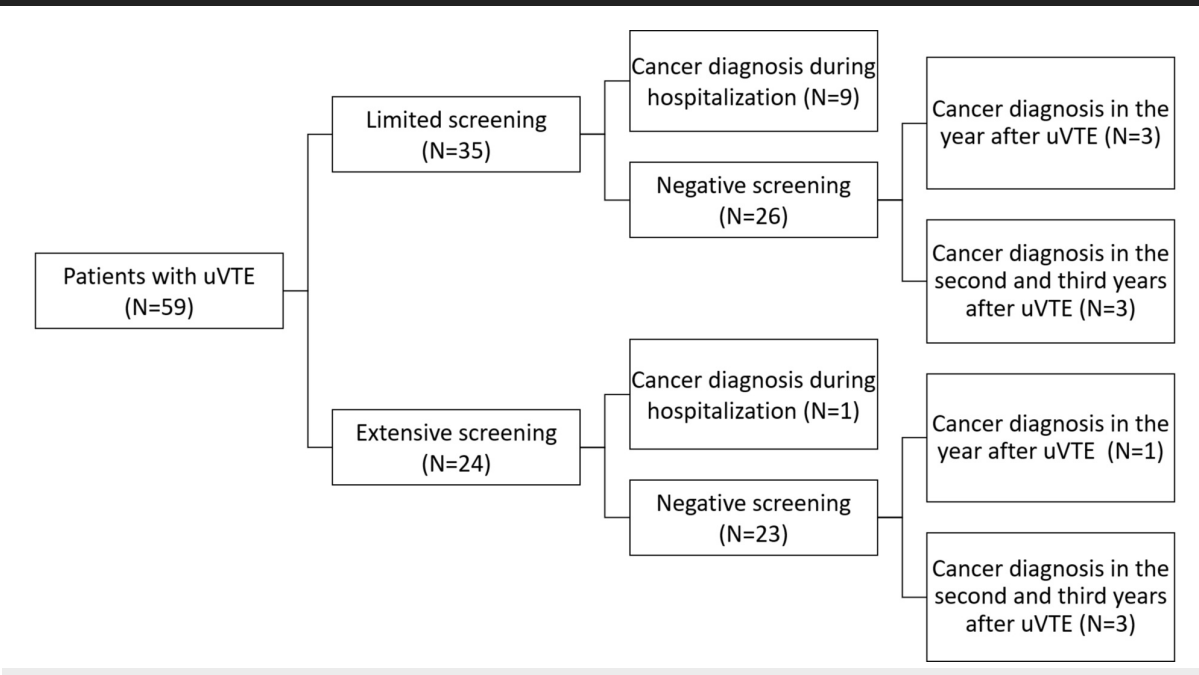

FIGURE 1: Scheme illustrating the results of screening for patients with unprovoked venous thromboembolism (UVTE)

There were ten inpatient diagnoses of cancer, nine of which in the subgroup that underwent LS. In the year following admission, four patients were diagnosed with cancer during the follow-up by their attending physicians: one patient had undergone ES and the remaining three LS. In the second and third year after hospitalization, six more patients were diagnosed, three in each group. In both groups, $60.0 \%$ of patients had cancer at an advanced stage at time of diagnosis. These data can be seen in Table 4 and Figure 1 . 


\section{Cureus}

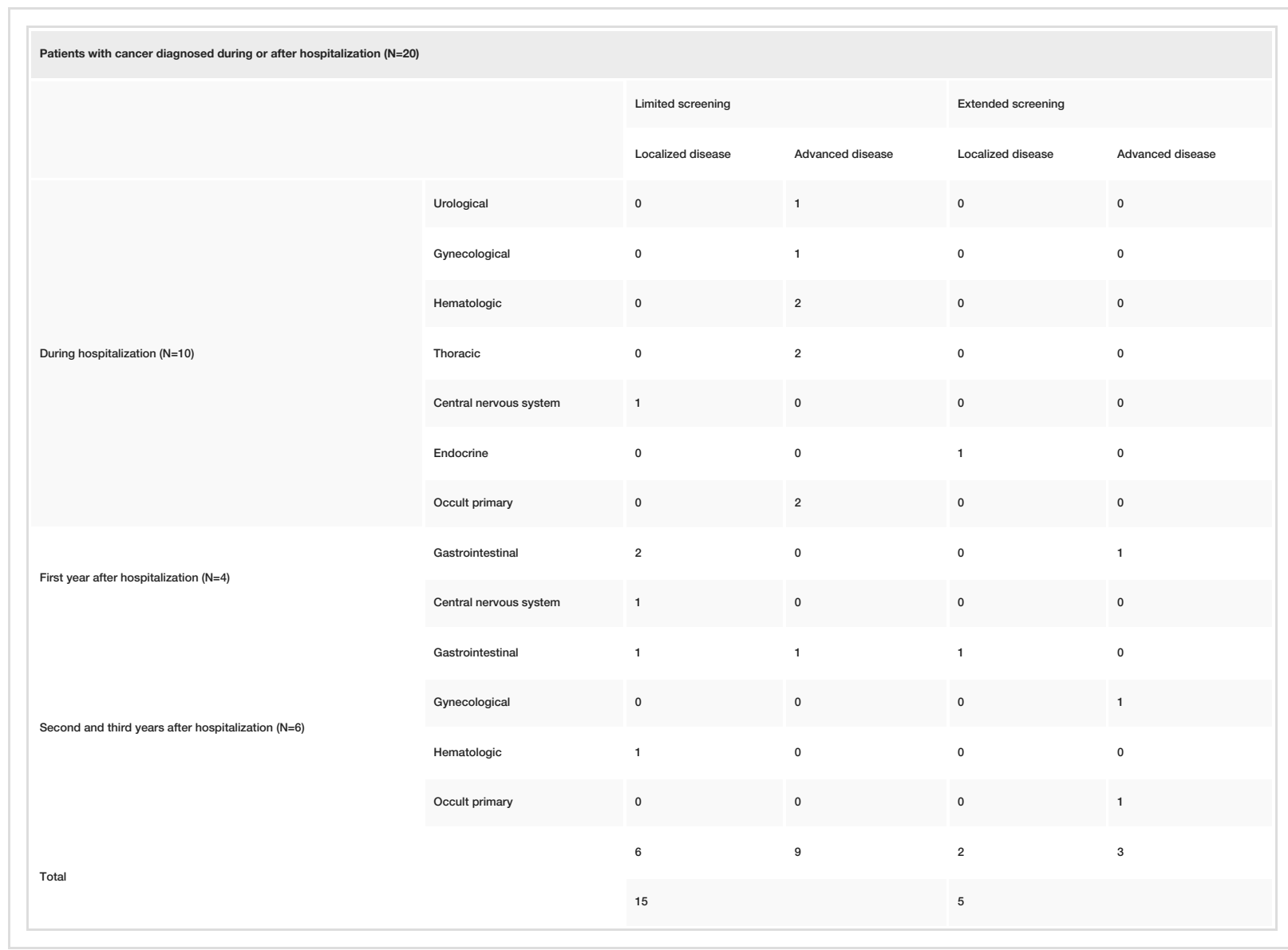

TABLE 4: Characteristics of patients with cancer diagnosed during or after hospitalization

Calculating the RIETE score for the population with UVTE, it is concluded that 41 patients had a score equal or above 3, of which 28 underwent ES. This subgroup included five patients who were diagnosed with cancer during hospitalization (only one who underwent ES) and four of those who were diagnosed in the following years and had undergone LS.

The rate of VTE recurrence was $7.3 \%(\mathrm{~N}=18)$, with more than half of the patients $(\mathrm{N}=10 ; 4.1 \%)$ presenting a diagnosis of cancer, already known or diagnosed during or after hospitalization. In-hospital mortality was $8.2 \%$, with eleven patients dying from cancer, two of whom were diagnosed during hospitalization (one from each screening group). There was no statistically significant difference in in-hospital mortality between the LS and ES groups: $2.9 \%$ vs. $4.2 \%$ ( $p=0.79$ ), respectively. Of the remaining eight patients diagnosed on admission, three died in the first year and two in the following 2-3 years. Of the ten patients diagnosed after hospitalization, six died in the following two years. There were also no statistically significant differences between mortality rates at one year $(8.6 \%$ vs. $8.3 \%$; $\mathrm{p}=0.97)$ and at three years $(20.0 \%$ vs. $20.8 \%$; $\mathrm{p}=0.94)$ between the LS and ES groups respectively.

\section{Discussion}

An occult cancer is detected in 4.5\%-5.0\% of patients with VTE and several studies show that LS diagnoses up to $90 \%$ of hidden neoplasms in patients with uVTE $[5,6,11,19]$. It was part of the main objective of this study to calculate the incidence rate of cancer diagnosis in hospitalized patients with VTE, which was 10 out of 245 (4.1\%) of the patients. In the inpatient regime, limited screening diagnosed $90 \%$ of the neoplasms (9 out of 10).

Screening techniques differ between trials, both in relation to LS, which only in some trials include screening tests related to patients age and gender, and in relation to ES, in which most trials include CT or PET/CT but others also include endoscopic exams. This fact makes it difficult to compare the data, but the conclusions are generally coincident. The Subsequent Diagnosis Of Malignancy in Patients presenting with Idiopathic Venous Thromboembolism (SOMIT) trial in 2004 showed that the difference in diagnoses between the two screening approaches was not statistically significant (10\% in LS and 14\% in ES) and that, although the diagnoses were made earlier in the ES group, this difference was not reflected in mortality $[8,12]$. Between 2011 and 2016, three trials were published comparing the two types of screening. The 
Trousseau trial compared LS with an ES including abdominal-pelvic CT and mammography, which was not associated with improved prognosis [12]. In the Screening for Occult Malignancy in Patients with Idiopathic Venous Thromboembolism (SOME) randomized trial, the number of cancer diagnoses did not increase by the addition of thoraco-abdominal-pelvic CT and tumour markers to the screening and there was no difference in time until diagnosis or mortality [9]. Similarly, Prandoni et al. concluded in a randomized, multicentre trial with 195 patients, that CT-based screening and faecal occult blood testing does not bring significant benefit over LS based on clinical criteria [10]. The Standard Diagnostic Procedures With or Without Fludeoxyglucose F 18 Positron Emission Tomography in Finding Cancer in Patients With a Blood Clot in a Vein (MVTEP) randomized trial is the largest to evaluate the inclusion of PET/CT in the screening, having shown that no more neoplasms were detected in a statistically significant percentage, but PET/CT had an important predictive value, with the risk of cancer being lower after negative PET/CT than after a negative LS $[2,20,21]$. A Cochrane review published in 2015 revealed that there was insufficient evidence that additional tests besides LS reduced morbidity or mortality [5,22]. A meta-analysis that included the 1,830 patients of Trousseau, SOME and MVTEP trials, concluded that ES was not effective in reducing mortality, which the authors associated with the reduced number of cancer diagnoses $(\mathrm{N}=98 ; 5.4 \%)$ and the heterogeneity of screening between tests and samples [6]. Another meta-analysis, by Zhou M. et al., with 2274 patients from five randomized trials, reached similar conclusions, with no difference in the risk of non-diagnosis or mortality between the two types of screening [11].

The main objective of our study was to compare the two types of screening by calculating the incidence of cancer (during hospitalization, in the first year and in the second and third years after) and assessing their impact on mortality. As this is a retrospective study and the tests performed depended on the decision of the attending medical team, the exams performed were not coincident among the various patients in each screening subgroup. However, the inclusion in the definition of LS or ES was similar with what was performed in most trials. There were no statistically significant differences between the rates of inpatient cancer diagnosis $(8.6 \%$ vs. $4.2 \%$; $=0.51)$ or in mortality rates during hospitalization $(2.9 \%$ vs. $4.2 \% ; \mathrm{p}=$ $0.79)$, at one year $(8.6 \%$ vs. $8.3 \%$; $=0.97)$ and three years $(20.0 \%$ vs. $20.8 \% ; p=0.94)$ between the LS and ES groups respectively. These results are compatible with other trials in which the median age and rate of detection of neoplasms in the advanced stage was similar to our analysis. Several trials concluded that patients diagnosed with cancer after uVTE had a poor prognosis, with most deaths occurring in the first year of follow-up. The authors of these trials raise the possibility that the association of VTE with cancer may reflect a more aggressive disease and which would probably become symptomatic soon, so an earlier diagnosis may not have an impact on the prognosis $[6,12]$.

The ideal follow-up time after an episode of uVTE is not yet fully established. The risk of diagnosing cancer is highest in the first six months and remains high for at least twelve months, but some studies show that it remains higher than in the general population up to six years after uVTE [3-6]. For this reason, in our study, cancer diagnosis data were collected up to one year and up to the third year after the uVTE episode. There were four diagnoses in the first year and six in the second and third years, which corresponds respectively to 6.8 and $10.2 \%$ of the population with UVTE, in which $40.0 \%(\mathrm{~N}=4)$ had undergone ES. These data raise the possibility that the risk of cancer might indeed remain high for more than the first year after the episode, but it is not possible to draw conclusions.

The cost-effectiveness analysis of including CT in the screening of the Trousseau and SOME trials showed that it is not cost-effective, as it requires more costs without bringing greater efficacy in detecting occult cancer than LS $[12,23]$. The post-hoc analysis of the cost-benefit of adding PET/TC to screening in the MVTEP trial, however, showed that this may be a valid option to consider since, although more expensive, screening with PET/TC lead to less late diagnoses and improved quality of life when compared to LS [24]. Since the screenings were not uniform in our study, it was not possible to carry out this analysis.

When calculating the RIETE score for the population with uVTE, we concluded that $69.5 \%(\mathrm{~N}=41)$ had a score equal or above 3 and, therefore, a risk of occult cancer superior to $10 \%$. According to the score validation trials, this may be the subgroup that benefits the most from ES [18]. In these trials, one-third of the population was at high risk, which leads to the conclusion that our population would have a higher risk. Of the 41 patients who had a score equal or above 3, only 28 underwent ES. In this group, were included 8 patients with a diagnosis of cancer during hospitalization or in the 1-3 years after who had undergone LS. These data raise the possibility that the approach to these patients could have been more appropriate if the RIETE score had been used to identify high-risk patients.

The main limitations of this study relate to the fact that it is a single-centre and retrospective study, which interferes in the study design and in the data collection, which was made based on computer records. The retrospective design makes it difficult to standardize the screenings in the study, as the exams were ordered by different medical teams at different times in the five-year period. The sample size is also small, which is an obstacle highlighted in several trials given the fact that only about $5 \%$ of patients with uVTE present a diagnosis of cancer.

A second point in the main objectives of this work was to develop a protocol for the approach of patients with uVTE. Thus, based on a literature review and this retrospective analysis, we suggest the protocol that can be observed in Figure 2. It is an objective that, depending on the approval of the protocol by the Internal 
Medicine Service of our hospital, it can be implemented, and a prospective study can be designed to analyse the results.

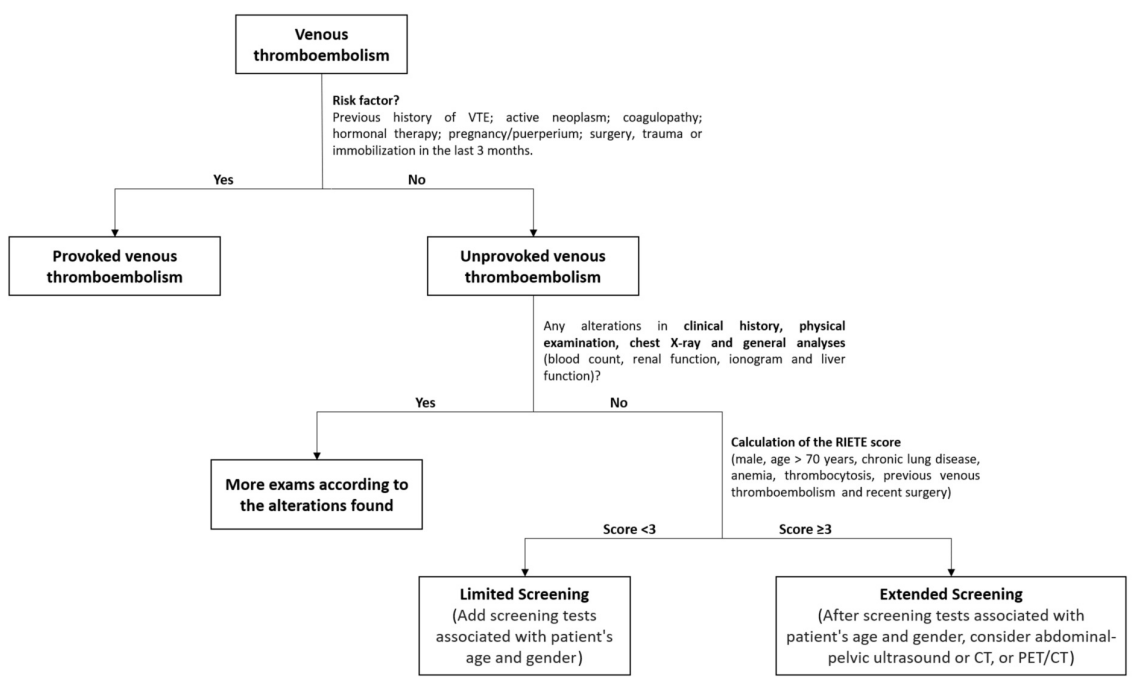

FIGURE 2: Protocol for the approach of patients with UVTE

CT: computed tomography; PET: positron emission tomography.

\section{Conclusions}

In this study, the incidence of cancer diagnosis in patients with VTE was $4.1 \%$ and there were no statistically significant differences in the rates of inpatient cancer diagnosis $(8.6 \%$ vs. $4.2 \%$; $=0.51)$ or in mortality rates during hospitalization ( $2.9 \%$ vs. $4.2 \%$; $=0.79)$, at one year after $(8.6 \%$ vs. $8.3 \%$; $=0.97)$ and at three years after $(20.0 \%$ vs. $20.8 \%$; $\mathrm{p}=0.94$ ) between the LS and ES groups respectively, with both results being similar to most of the literature. When calculating the RIETE score, which predictive value is validated for the population with uVTE, we concluded that $69.5 \%(\mathrm{~N}=41)$ had a score equal or above 3 , which is equivalent to a risk of occult cancer superior to $10 \%$. It is important that scores like this are included in clinical practice and that future studies focus on identifying subgroups that may benefit from more intensive screening strategies, in order to increase the number of diagnoses without increasing the costs and the number of futile procedures.

\section{Additional Information \\ Disclosures}

Human subjects: Consent was obtained by all participants in this study. Animal subjects: All authors have confirmed that this study did not involve animal subjects or tissue. Conflicts of interest: In compliance with the ICMJE uniform disclosure form, all authors declare the following: Payment/services info: All authors have declared that no financial support was received from any organization for the submitted work. Financial relationships: All authors have declared that they have no financial relationships at present or within the previous three years with any organizations that might have an interest in the submitted work. Other relationships: All authors have declared that there are no other relationships or activities that could appear to have influenced the submitted work.

\section{References}

1. Bauer KA, Lip GYH: Evaluating adult patients with established venous thromboembolism for acquired and inherited risk factors. UpToDate, 2019.

2. Robin P, Le Roux P, Le Moigne E, et al.: Additional testing following screening strategies for occult malignancy diagnosis in patients with unprovoked venous thromboembolism. Thromb Res. 2017, 155:6-9. 10.1016/j.thromres.2017.04.022

3. Sun LM, Chung WS, Lin CL, Liang JA, Kao CH: Unprovoked venous thromboembolism and subsequent cancer risk: a population-based cohort study. J Thromb Haemost. 2016, 14:495-503. 10.1111/jth.13251

4. Dhakal P, Gundabolu K, Bhatt V: An algorithmic approach to management of venous thromboembolism . Clin Appl Thromb Hemost. 2017, 23:511-517. 10.1177/1076029616652727

5. Husseinzadeh $\mathrm{H}$, Carrier M: Occult cancer detection in patients with hemostatic disorder and venous thromboembolism. Thromb Res. 2018, 163:242-245. 10.1016/j.thromres.2017.05.034

6. Robin P, Otten HM, Delluc A, et al.: Effect of occult cancer screening on mortality in patients with unprovoked venous thromboembolism. Thromb Res. 2018, 171:92-96. 10.1016/j.thromres.2018.09.055

7. Gheshmy A, Carrier M: Venous thromboembolism and occult cancer: impact on clinical practice . Thromb 
Res. 2016, 140:S8-S11. 10.1016/S0049-3848(16)30091-3

8. Piccioli A, Lensing AWA, Prins MH, et al.: Extensive screening for occult malignant disease in idiopathic venous thromboembolism: a prospective randomized clinical trial. J Thromb Haemost. 2004, 2:884-9. 10.1111/j.1538-7836.2004.00720.x

9. Carrier M, Lazo-Langner A, Shivakumar S, et al.: Screening for occult cancer in unprovoked venous thromboembolism. N Engl J Med. 2015, 373:697-704. 10.1056/NEJMoa1506623

10. Prandoni P, Bernardi E, Valle FD, et al.: Extensive computed tomography versus limited screening for detection of occult cancer in unprovoked venous thromboembolism: a multicenter, controlled, randomized clinical trial. Semin Thromb Hemost. 2016, 42:884-890. 10.1055/s-0036-1592335

11. Zhou M, Zhang L, Ding Y, et al.: Extensive screening for occult malignancy in unprovoked venous thromboembolism: a meta-analysis. Thromb Res. 2017, 157:147-153. 10.1016/j.thromres.2017.07.019

12. Keeling D, Klok FA, Le Gal G: Controversies in venous thromboembolism - 2015 . Blood Rev. 2016, 30:27-33. 10.1016/j.blre.2015.07.003

13. Khorana A, Carrier M, Garcia D, Lee AY: Guidance for the prevention and treatment of cancer-associated venous thromboembolism. J Thromb Thrombolysis. 2016, 41:81-91. 10.1007/s11239-015-1313-4

14. Han D, Hartaigh B, Lee JH: Impact of D-dimer for prediction of incident occult cancer in patients with unprovoked venous thromboembolism. PLoS One. 2016, 11:e0153514. 10.1371/journal.pone.0153514

15. Bertoletti L, Robin P, Jara-Palomares L, et al.: Predicting the risk of cancer after unprovoked venous thromboembolism: external validation of the RIETE score. J Thromb Haemost. 2017, 15:2184-7. 10.1111/jth.13842

16. Robin P, Le Roux PY, Tromeur C, et al.: Risk factors of occult malignancy in patients with unprovoked venous thromboembolism. Thromb Res. 2017, 159:48-51. 10.1016/j.thromres.2017.08.021

17. Ihaddadene R, Corsi DJ, Lazo-Langner A, et al.: Risk factors predictive of occult cancer detection in patients with unprovoked venous thromboembolism. Blood. 2016, 16:2035-2037. 10.1182/blood-2015-11-682963

18. Jara-Palomares L, Otero R, Jimenez D, et al.: Validation of a prognostic score for hidden cancer in unprovoked venous thromboembolism. PLoS One. 2018, 13:e0194673. 10.1371/journal.pone.0194673

19. van Es N, Le Gal G, Otten HM, et al.: Screening for occult cancer in patients with unprovoked venous thromboembolism: a systematic review and meta-analysis of individual patient data. Ann Intern Med. 2017, 167:410-417. 10.7326/M17-0868

20. Robin P, Le Roux PY, Planquette B, et al.: Limited screening with versus without ${ }^{18} \mathrm{~F}$-fluorodeoxyglucose $\mathrm{PET} / \mathrm{CT}$ for occult malignancy in unprovoked venous thromboembolism: an open-label randomised controlled trial. Lancet Oncol. 2016, 17:193-199. 10.1016/S1470-2045(15)00480-5

21. Robin P, Carrier M: Revisiting occult cancer screening in patients with unprovoked venous thromboembolism. Thromb Res. 2018, 164:S7-S11. 10.1016/j.thromres.2017.12.024

22. Robertson L, Yeoh SE, Stansby G, Agarwal R: Effect of testing for cancer on cancer and venous thromboembolism (VTE)-related mortality and morbidity in patients with unprovoked VTE. Cochrane Database Syst Rev. 2017, CD010837. 10.1002/14651858.CD010837.pub3

23. Coyle K, Carrier M, Lazo-Langner A, et al.: Cost effectiveness of the addition of a comprehensive CT scan to the abdomen and pelvis for the detection of cancer after unprovoked venous thromboembolism. Thromb Res. 2017, 151:67-71. 10.1016/j.thromres.2017.01.007

24. Robin P, Kumar S, Salaun PY, et al.: In patients with unprovoked VTE, does the addition of FDG PET/CT to a limited occult cancer screening strategy offer good value for money? A costeffectiveness analysis from the publicly funded health care systems. Thromb Res. 2018, 171:97-102. 10.1016/j.thromres.2018.09.050 